(C)2009 IEEE. Personal use of this material is permitted. However, permission to reprint/republish this material for advertising or promotional purposes or for creating new collective works for resale or redistribution to servers or lists, or to reuse any copyrighted component of this work in other works must be obtained from the IEEE. 


\title{
A State Of The Art Opinion Mining And Its Application Domains
}

\author{
Haji Binali, Vidyasagar Potdar, Chen Wu \\ Digital Ecosystems and Business Intelligence Institute, \\ Curtin University of Technology, Australia \\ hbinali@yahoo.com v.potdar@curtin.edu.au chen.wu@curtin.edu.au
}

\begin{abstract}
This paper critically evaluates existing work, presents an opinion mining framework and exposes new areas of research in opinion mining. Individuals, businesses and government can now easily know the general opinion prevailing on a product, company or public policy. At the core of this field is semantic orientation of subjective terms in documents or reviews which seeks to establish their contextual connotation through opinion mining. Overall item sentiment can be expressed based on its sentiment words in general or by specifically identifying its features and the opinions being expressed about them. This leads us to the motivation of the framework for opinion mining and categorizing current literature in such a manner as to make clear, research opportunities. The freedom offered by the web as a platform for presenting opinions on any subject brings with it many new opportunities.
\end{abstract}

Keywords: Opinion mining, supervised learning, unsupervised learning, data mining

\section{INTRODUCTION}

Industry or manufacturing companies that produce new products want to know how their customers feel about these products and this information can be acquired by studying opinions from review portals [2]. At the same time, users or consumers want to know which product to buy so they also read reviews and try to make their decisions. It has been seen that online opinions are getting popular day by day and these opinions represent wealth of information which can be beneficial for the industry as well as consumers [1].

However doing this manually is a mundane and time consuming job. As an example, manufacturing organizations prefer information in a format that is easier to use, so automating this process is very useful [3].This is where opinion mining comes to picture. In the web, opinions can be expressed in the form of text, image, audio or video data. This paper is biased towards text mining as this is a widely researched area. Opinion mining can be defined as a sub discipline of computational linguistics that is concerned with the opinion that a document expresses $[4,5]$. Sentiment classification is about determining the subjectivity, polarity (positive/negative) and polarity strength (weakly positive, mildly positive or strongly positive) of an opinion text $[2,6]$. Various terms have been used by researchers to define sentiment classification: opinion mining, sentiment analysis, sentiment extraction or affective rating [6] . For simplicity purposes in this paper, our use of the term, opinion mining, encompasses all these meanings.
Opinion mining aims to extract opinions from information sources (user generated content or user generated media) such as reviews, and present them to the users in a user friendly manner (graphically for example). Since this field is very new and much work is currently being done in this area, we take the opportunity to categorize a significant part of the existing work in a way that can help researchers to study this field in further detail.

This paper is organized in the following sections. Section II shows closely related fields of opinion mining whose fundamental understanding is crucial. We present an in depth discussion of a classification framework based on existing work in section III. Section IV shows application domains for opinion mining. We conclude our paper with section V. 


\section{COUSINS: CLOSELY RELATED FIELDS OF OPINING MINING}

Information Extraction (IE): Is the transformation of unstructured textual information into a structured format that is commonly stored in databases and can be used for data mining purposes based on machine language learning. Specific data is extracted from corpora and fits into an existing template. This improves the precision of the retrieved information and can be used as basis for categorizing the extracted data. Named Entity Recognition (NER) is a method by which named entities are identified in text. It can be used as a prerequisite for information extraction and improves information retrieval by indexing and querying databases.

Information Retrieval (IR): The search for information is usually based on a query. Common information retrieval systems include popular search engines such as Google, yahoo search, Live search and AltaVista. Access to books and other documents at Universities and public libraries is also facilitated by IR systems. According to our understanding of the literature, the major distinction between IE and IR is that IR provides more precise data by querying databases based on a topic.

Natural Language Processing (NLP): This refers to the processes that computers use to convert human language (written/spoken) into useful or practical knowledge that a computer may understand and use while interacting with other computers. NLP involves processing text using lexical, syntactic and semantic knowledge. NLP is also a sub-discipline of artificial intelligence.

Machine Language Learning (ML): Machine language learning or simply machine learning (ML), refers to processes that involve the building and evolution of machine dictionaries that model human behavior, thoughts and responses. There are various data mining tasks that can be performed, supervised learning (classification), unsupervised learning (clustering), association rule mining and sequential pattern mining. In this paper, we will look at the common ones; supervised learning and unsupervised learning.

Web Data mining: We introduce data mining as it has been widely applied in opinion mining tasks. Data mining is also called data or knowledge discovery in databases. It is discovery of useful knowledge from data sources such as databases and the web. It involves various fields like machine learning, statistics, databases, artificial intelligence, information retrieval and data visualization.

\section{OPINION MINING OVERVIEW}

Fig. 1 shows an overview of the processes in opinion mining. It is logically derived from a critical analysis of existing research in opinion mining. We note that there are two main methods by which an opinion can be extracted. Naturally, the first step is to know the item for which an opinion is required through item extraction. This only gives an overall positive/negative opinion about the item, without any specific details of what is actually being considered. Nothing is known about the pros and cons of the features. However, to know the item sentiment, based on its feature sentiments, feature extraction must be performed. This enables one to differentiate between good and bad features.

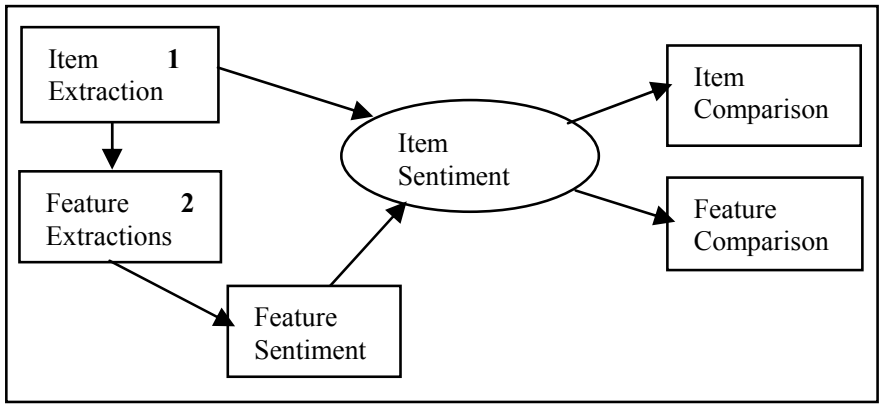

Fig. 1 Opinion mining framework

Whereas in step 1, although it can be known that item A is $\mathrm{good} / \mathrm{bad}$, there is no apparent justification for this. However, in step 2, it can be known why item $\mathrm{A}$ is good/bad. Consider Camera A as an example, whereas step 1 can inform us that the camera is $\mathrm{good} / \mathrm{bad}$, step 2 can inform us about the zoom functionality, battery-life, weight and size of the camera. Based on this further detail, a more subjective opinion can be deduced. Interestingly, we note that researchers have largely focused their efforts on item sentiment but minimal research has been carried out on item extraction.

Furthermore, as shown in Fig. 1, the output for item sentiment can be used as the input for item comparison and feature comparison. Fig. 1, shows us that a reviewer can have the option of comparing item A with item B or the features of item A with those of item B. In the remainder of this section, we present an in depth study of the 6 areas in the framework.

\section{A. Item Extraction}

As used within the context of this paper, the terms: entity and object have the same connotation as item. The Item name is instrumental in knowing the application domain for a search term. Item extraction refers to the object being mentioned in the review e.g. Camera, Mobile Phone, MP3 player, Notebook Computer or Movie. We consider item extraction to be critical for extracting opinions because it helps us to know the item whose opinion is being extracted. Unfortunately, minimal literature is available in this area. However, [7] provide comprehensive literature on the unsupervised extraction of web entities. 


\section{B. Feature Extraction}

This approach takes a step further than Item-based approach in terms of pursuing finer granularity of opinion. The basic motivation of feature-based approach is that a negative customer opinion on a product does not necessarily mean this customer dislikes every aspect of this product, and vice versa. This approach models a product consisting of a number of subcomponents, each of which is associated with a set of attributes that can be evaluated through opinion expressions. Note that feature represents both components and attributes. Consider a digital camera, which has several features as illustrated in [1]: picture quality, battery life, zoom, size, weight. For example, a camera with poorer picture quality may have a very long battery life and light weight. A very important step in feature-based approach is to extract features for different products and services.

Feature extraction is an activity that can be carried out after item extraction so that it may be known why an item has be categorized as thumbs up or thumbs down. Rather than just establishing semantic orientation of the terms used for expressing an opinion and extracting an opinion based on that, feature extraction goes a level lower than item extraction by looking for the item features whose opinion can be used to extract the feature sentiment. It must be stated here that although some work has been carried out in this area, it is not as comprehensive as item sentiment, but definitely more comprehensive than what has been done for item extraction. Some notable work has been done by [8] who formally defined and modeled the process of feature extraction for opinion mining.

\section{Feature Sentiment}

Feature sentiment is the opinion that is expressed for an item based on its features. After the features have been identified, a feature sentiment can be expressed for each feature, which tells us something about the weaker and finer points of an item's features. For example, long battery life, portable size, beautiful colors, excellent cast, poor actors. A point of interest here is the case of the movie review. Our research shows that it is hard for current algorithms to distinguish between opinions of the movie in general and those of the actors. In some instances, a movie may get a positive review because a popular actor is involved but the movie itself may be uninteresting. With scenarios of this nature, it becomes necessary to differentiate between reviews of the movie and those of the actors. [9] Have managed to develop an algorithm that can be used to extract the feature sentiments for reviews in Japanese, thus acting as a precedent for future research on language independent, feature sentiment extraction algorithms

\section{Item Sentiment}

This refers to the overall sentiment being expressed on the object, for example, recommended/un-recommended, good/bad, buy/don't buy, excellent film/ boring film. This is very useful were a prevailing opinion needs to be quickly known. A bulk of the literature is concentrated towards finding out the item sentiment. Researchers have shown a lot of interest in this area. Notably, Turney [10] presented a popularly used paradigm which provides a basis for extracting the opinion of an item. The terms used in the review are assumed to be subjective and can be divided into one of three groups, positive, negative or neutral. Most research has focused on weighing the subjectivity of positive and negative terms to extract the review sentiments while ignoring neutral ones. However, other researchers believe that neutral (objective) terms must be taken into consideration and can improve the accuracy of results.

\section{E. Item Comparison}

Item comparison depicts a finer granularity of an opinion between Entity $\mathrm{A}$ and other entities, for example, camera $\mathrm{AB}$ vs. camera $\mathrm{XY}$. AB cameras tend to have more positive reviews than XY Cameras. This is important to shoppers who may not have time to shop around for the best price and product to buy. Shoppers can save a lot of time in decision making processes for mundane items, thereby making a double saving in terms of both time and money. [9] have developed a novel way of graphically depicting comparisons.

\section{F. Feature Comparison}

Feature comparison is for comparison between Entity A and other entities. As an example, one may want to find out how the features of a PQ camera compare with those of a XY Camera. Following is an example of feature comparison; the PQ camera tends to have a longer battery life and superior zoom functionality than camera XY. Some innovations that have taken place in this area involve graphically displaying all the features of a product. Undoubtedly, this adds to the shopping experience by displaying all the pros and cons between item features. Furthermore, manufacturers can easily identify features that are not meeting consumer expectations and needs.

\section{OPINION MINING APPLICATION DOMAINS}

Opinion mining can be used in various fields to meet varied purposes. In this section, we take the opportunity to present some of the common ones. The examples presented in this section are not exhaustive but simply a snap shot of the possibilities.

\section{A. Shopping}

Perhaps the most popular use of opinion mining is decision support for consumers. Consumers are actively involved in comparison shopping over the internet. Popular web sites like amazon.com allow customers to express their opinions on their websites. Customers can easily view the opinions for products and identify how features between products compare with each other. In some instances, after an opinion has been mined and processed, knowledge is presented to the user in a graphical format for easy comparison of product features. We will 
consider the comments on an electronic product from an online shop.

"I needed a high-powered laptop for my business needs. Dell offered a variety of products that met my requirements. Their product information was concisely and completely explained, which made my selection process very easy. Their website was easy to use and attractively presented. The merchandise was delivered on time, as per their promise. Shipping charges were very reasonably priced. I was able to take care of all my needs online, thereby not having to use their telephone support service. Their online help and support was excellent. I would recommend Dell Small Business to anyone. [11]”.

We note that the opinion is about Dell Small Business and the various features being talked about are delivery time, shipping charges, support and web site navigation.

\begin{tabular}{|c|c|}
\hline $\begin{array}{l}\text { Online Shopper } \\
\text { Jul 24,2008 }\end{array}$ & Dout of 0 people found this review helpful. \\
\hline $\begin{array}{l}\text { The worst custome } \\
\text { - Calling me twice } \\
\text { wanted to cancel t } \\
\text { anyone. (more) }\end{array}$ & $\begin{array}{l}\text { poorly handled from start to finish } \\
\text { anging up on me because I } \\
\text { recommend this business to }\end{array}$ \\
\hline
\end{tabular}

Quite a dissapointment.

by derekrubin, May 1008

Pros: A solid device for what it is, a fairly interesting new interface, vivid screen Cons: The back casing loves fingerprints and scratches, video feature shoved down throat

I have owned a number of different iPods including all of the generations of the Nano, the Mini, and the $30 \mathrm{gig}$ iPod Video. This Third-Generation Nano is all in all, my least favorite (with the Second-Generation Nano my favorite). It seems strange

Read the full review

\section{B. Entertainment}

Movie goers and home TV viewers can quickly access the opinion on recent releases and popular movies and programs. Currently, there is the internet movie database (IMDB) which provides online reviews for movies as well as TV programs. This acts as a guide for people who are unsure about which movies to watch. We present an extract from the IMDB. "Christopher Nolan's second bundle of joy "The Dark Knight" EXCEEDED all of my expectations!!! I can HONESTLY tell you that: as good as Jack Nicholson was in Batman'89 he is CHILD'S PLAY compared to this Joker. He is sadistic, psychotic, and downright SCARIER and PSYCHOLOGICALLY disturbing than the previous incarnation of The Clown Prince of Crime and Ledger gives it his all to do him justice. The action is great, and the plot is deeper and engrossing. [11]"

From the previous opinion, we observe that capital letters and exclamation signs are being used to emphasize emotions. Furthermore, we observe that the first comment is positive and refers directly to the movie, "The Dark Knight". However, subsequent statements refer to the actors and it is their attributes that are being mentioned. The last statement mentions the film once again. This kind of opinion, which revolves between the actors and the movie, is relatively simple for a human reader to understand but not so for a machine. Therefore, this presents some complexity to machine language learning. It is evident that two objects are being described, the movie and actors. Although words with a negative connotation (sadistic, psychotic, and disturbing) are being used to express positive aspects of the movie, it does not mean that the film is not highly recommended but rather, just an illustration of the complexity that exists for machine language learning. Moreover, most opinions about movies are expressed in this way.

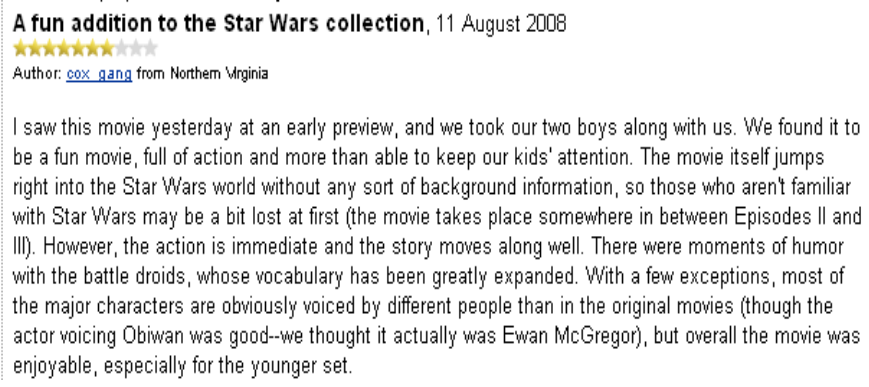

I saw this movie yesterday at an early preview, and we took our two boys along with us. We found it to be a fun movie, full of action and more than able to keep our kids' attention. The movie itself jumps right into the Star Wars world without any sort of background information, so those who aren't familiar with Star Wars may be a bit lost at first (the movie takes place somewhere in between Episodes II and III). However, the action is immediate and the story moves along well. There were moments of humor with the battle droids, whose vocabulary has been greatly expanded. With a few exceptions, most of the major characters are obviously voiced by different people than in the original movies (though the actor voicing Obiwan was good--we thought it actually was Ewan McGregor', but overall the movie was enjoyable, especially for the younger set.

\section{Government}

Governments can mine the prevailing opinions on public policy. Election candidates can become more knowledgeable about specifics of the opinion poll. This knowledge can assist politicians to identify where their strengths and weaknesses lie according to their electorate. Consider the following political opinions that have been expressed. "Expect more inflation. More unemployment. Really, we need some better selection process. Who chooses these people? They make history by raising rates for the first time in the lead up to an election. I have no confidence in the published figures." [12]

A quick glance at these terms indicates a sense of dissatisfaction among the electorate. Furthermore, key areas of concern are addressed in terms of what is lacking and what the expectations are.

Issues that deal with public policy normally categorize voters into one of three groups, for, against or neutral. A good example is the statement, "I think this all seems extremely harsh. Boredom, if anything, is a sign of intelligence." [12] A statement of this kind makes it clear that the opinion is for the motion. The advantage of opinion mining over traditional opinion polls like telephone polls is that it can be determined why electorates are for or against a proposal. Most web sites, particularly those whose fundamental objective is to provide news, have a facility for web users to express their opinions on their websites. 


\section{Research and Development}

Product reviews can be used by manufacturing companies to improve features and provide a platform for innovation. Web based applications could offer platforms for customers to design products and submit the designs to the manufacturing companies. An approach of this nature could significantly assist in establishing features that are liked by customers. Consider the following review for an electronic product, "The click wheel is HORRIBLE and completely lacks response and sensitivity." [13]

This is a negative opinion being expressed about the click wheel. The use of upper caps signifies to the reader the extent of disappoint. If opinion mining is able to detect emotions of this kind being expressed in evaluative text, it will prove to be very beneficial. This will act as an indicator on how the product has been received by a consumer. However, after expressing negative opinions on the product features, a statement such as "although I am really disappointed this is probably still the best high capacity music player on the market [13]". This positive statement indicates to the R \& D department and marketing departments that the music player is still the best in the market and it is the high capacity which is favored by customers. Further examples can be obtained from web sites like bizrate.com and epinion.com.

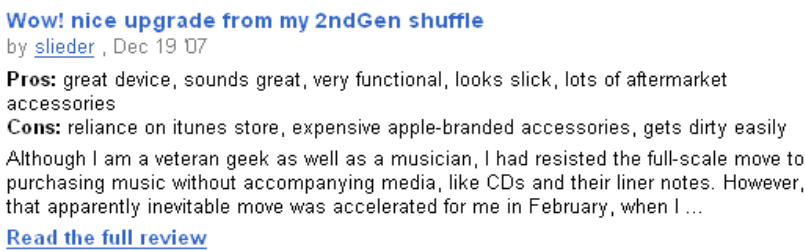

\section{E. Marketing}

Companies can now make savings on marketing expenses by requesting for reviews on their websites and peer review websites. This eliminates the need for business consultants to conduct surveys as companies can now have all the data they need online. The advent of the internet has brought along with it new ways of marketing. We present viral marketing as an example. Viral marketing is the use of social networks to spread product and service information. With the advent of the internet, social networks such as MySpace, Facebook and Hi5 are offering a new platform for information exchange. Family and friends can now recommend products/services to each other or seek more knowledge about a product or service before committing themselves. It is analogous to the traditional word of mouth marketing of products and services. To encourage postings and recommendations among peers, marketers normally offer incentives like discounts for recommendations that turn into purchases [14]. Let us consider the following recommendation for a tourism resort, "It is a land of contrasts and majesty, Africa at its most wild and unexplored [12]." Positive opinions of this kind can improve a products rating. WAYN (where are you now) is a social network that lets friends track each other during their travels, posting photographs and describing their adventures and experiences.

\section{F. Education}

In e-learning systems, user's opinions can be used to evaluate academic institutions and academics. Academics can know the sentiment on courses based on sentiment analysis of opinions expressed by students. This can help to improve service delivery and bolster marketing campaigns. Unit coordinators can know what students think about their team members and tutors by requesting them to provide online reviews as a part of course requirement. Curtin University of Technology offers units in which students must submit weekly peer reviews and also offers discussion forums. Such frameworks act as rich sources of user generated content that can be mined. Research findings by [15] reveal that e-learning systems adoption by tertiary institutions is still in its early phases. Members of the legal fraternity use legal blogs (blawgs) to express opinions. According to [16], students post their experiences online and law professors provide comprehensive analysis of court cases and post their findings to the community. Legal researchers can benefit from different opinions that are posted for a legal issue. Let us consider a sample opinion from an academics point of view.

"My research is improving my analytical, problem solving skills and ability to plan my own work. The feedback from the supervisor is valuable. The computing facilities are excellent. However, the monthly down load quota is too low for conducting research without being exceeded. [17]",

The overall opinion is for research (object) and it is positive. The features to extract an opinion on would be the supervisor, computing facilities and download quota. When data from different institutions of this kind is recorded and made readily available, it can be used for comparison purposes.

\section{Conclusion}

This paper presents the current state of the art in opinion mining. On the one hand, we discover that research has been concentrated towards finding out the sentiment on an item and classifying it as thumbs up or down. On the other hand, little research has been undertaken towards web queries that provide knowledge about an explicit item and its comparison with other items. Although reviewers would benefit more from a comparison between items and/or item features, our research findings reveal a different reality. To the best of our knowledge, minimal research has been done in this area. Opinion mining has many application domains including science \& technology, entertainment, education, politics, marketing, accounting, law, research and development. We hope that researchers will explore these areas in the future. 


\section{REFERENCES}

[1] M. Hu and B. Liu, "Mining Opinion Features in Customer Reviews," presented at 19th National Conf. on Artificial Intelligence, 2004.

[2] Wilson T, "Just how mad are you? Finding strong and weak opinion clauses," presented at In Proc. 19th National Conference on Artificial Intelligence. 2004.

[3] L. B. Hu M, "Mining and summarizing customer reviews.," presented at Proceedings of the tenth ACM SIGKDD international conference onKnowledge discovery and data mining 22:2525, 2004.

[4] A. Esuli and F. Sebastiani, "Determining Term Subjectivity and Term Orientation for Opinion Mining," presented at Proceedings the EACL 2006, 2006.

[5] S. F. Esuli A, "SENTIWORD NET : A Publicly Available Lexical Resource for Opinion Mining " presented at Proceedings of LREC, 2006, 2006

[6] S. F. Esuli A, "Determining the semantic orientation of terms through gloss classification," presented at Proceedings of the 14th ACM international conference on Information and knowledge management, Bremen, Germany 2005.

[7] O. Etzioni, M. Cafarella, D. Downey, Popescu A, T. Shaked, S. Soderland, D. Weld, and A. Yates, "Unsupervised named-entity extraction from the Web: An experimental study," 2004.

[8] M. Hu and B. Liu, "Mining and summarizing customer reviews.," presented at Proceedings of the tenth ACM SIGKDD international conference on Knowledge discovery and data mining 22:2525, 2004.

[9] G. Wang and K. Araki, "An Unsupervised Opinion Mining Approach for Japanese Weblog

Reputation Information Using an Improved SO-PMI Algorithm," IEICE TRANS. INF. \& SYST, vol. VOL.E91-D, pp. 1032-1041, 2008.

[10] Turney P, "Thumbs Up or Thumbs Down? Semantic Orientation Applied to Unsupervised

Classification of Reviews.," presented at In Proc. of the Meeting of the Association for ComputationalLinguistics (ACL'02), 2002.

[11] The Dark Knight, 2008. Retrieved from http://www.imdb.com/title/tt0468569/

[12] The Australian, 2008. Retrieved from http://www.theaustralian.news.com.au/politics/opinion/

[13] tanzaniatouristboard, 2008. Retrieved from http://tanzaniatouristboard.com/

[14] J. LESKOVEC, A. L. ADAMIC, and B. A. HUBERMAN, "The Dynamics of Viral Marketing," presented at ACM Transactions on the Web (TWEB) 2007.

[15] I. Elgort, "E-learning adoption: Bridging the chasm," presented at ascilite 2005 conference proceedings, 2005.

[16] J. Conrad and F. Schilder, "Opinion Mining in Legal Blogs," presented at Proceedings of the 11th international conference on Artificial intelligence and law 2007.

[17] Curtin Surveys 2008. Retrieved from http://planning.curtin.edu.au/mir/cass.cfm

[18] J.Dickinson, 2006.2 Retrieved from http://www.goodgearguide.com.au/index.php/taxid;2136212594;pid ;1713;pt;1
Bizrate.com, 2008. Retrieved from http://www.bizrate.com/ratings_guide/cust_review_detail_mid-27622 mr type--1 review id--

\section{$12145 \overline{17} 07 \overline{9} 9683800036200280101022273 . \mathrm{html}$}

[20] T. Wilson and J. Wiebe, "Annotating opinions in the world press," presented at In Proceedings of the 4th ACL SIGdial Workshop on Discourse and Dialogue (SIGdial-03), 2003.

[21] T. Cheng, X. Yan, K. Chen, and C. Chang, "EntityRank: Searching Entities Directly and Holistically ${ }^{\mathrm{C}}$."

[22] G. Carenini, R. Ng, and E. Zwart, "Extracting Knowledge from Evaluative Text," Third International Conference on Knowledge Capture, pp. pp. pp. $11-18,2005$.

[23] M. Popescu and O. Etzioni, "Extracting Product Features and Opinions from Reviews," presented at presented at Conference on Empirical Methods in Natural Language Processing, 2005.

[24] K. Kanayama and T. Nasukawa, "Fully Automatic Lexicon Expansion for Domain-oriented Sentiment Analysis," presented at kanayama-nasukawa:2006:EMNLP, 2006.

[25] T. Wilson, J. Wiebe, and R. Hwa, "Just how mad are you? Finding strong and weak opinion clauses," presented at In Proc. 19th National Conference on Artificial Intelligence., 2004.

[26] J. R. Martin and P. R. R. White, The Language of Evaluation: Appraisal in English. London: Palgrave, 2005.

[27] L. W. Ku and H. H. Chen, "Mining Opinions from the Web: Beyond Relevance Retrieval " Journal of the American Society for Information Science and Technology, vol. 58, pp. 1532-2882, 2007.

[28] K. Dave, S. Lawrence, and D. Pennock, "Mining the Peanut Gallery: Opinion Extraction and Semantic Classification of Product Reviews.," presented at In Proc. of the 12th Intl. World Wide Web Conference (WWW'03), 2003.

[29] L. W. Ku, Y. T. Liang, and H. H. Chen, "Opinion Extraction, Summarization and Tracking in News and Blog Corpora," presented at AAAI-CAAW, 2006

[30] D. Song, H. Lin, and Z. Yang, "Opinion Mining in e-Learning System," presented at Network and Parallel Computing Workshops, 2007. NPC Workshops. IFIP International Conference on, Liaoning, 2007.

[31] B. Liu, M. Hu, and J. Cheng, "Opinion Observer: Analyzing and Comparing Opinions on the Web," presented at presented at 14th International World Wide Web Conference, 2005, 2005.

[32] B. Liu, M. Hu, and J. Cheng, "Opinion Observer: Analyzing and Comparing Opinions on the Web," presented at Proceedings of the 14th international World Wide Web conference Chiba, Japan, 2005.

[33] B. Pang, L. Lee, and S. Vaithyanathan, "Sentiment Classification Using Machine Learning Techniques.," 2002.

[34] K. C. C. Chang and B. He, "Statistical Schema Matching across Web Query Interfaces.," presented at In Proc. of the 2003 ACM SIGMOD Intl. Conf. on Management of Data(SIGMOD’03, 2003.

[35] C. Whitelaw, G. Navendu, and A. Shlomo, "Using Appraisal Taxonomies for Sentiment Analysis," presented at Proceedings of the 14th ACM international conference on Information and knowledge management 2005. 